\title{
Correction: Risk of poultry compartments for transmission of Highly Pathogenic Avian Influenza
}

\section{The PLOS ONE Staff}

There are multiple formatting errors in Table 2. Please see the complete, correct table here.

Table 2. The risk of HPAI transmission jumps from one area to another, due to a compartment with only egg transports (i.e. no animal transports), and with no compartment farms situated in a DPPA.

\begin{tabular}{l|l}
\hline Quantity & Value \\
\hline Ratio $^{1)}$ & $0.0015(0.0013-0.0017)$ \\
\hline 'Single-event' transmission probability ${ }^{2)}$ & $1.210^{-6}-1.610^{-5}$ \\
\hline 'Triple-event' transmission probability ${ }^{2)}$ & $3.610^{-10}-5.110^{-8}$ \\
\hline & \\
&
\end{tabular}

https://doi.org/10.1371/journal.pone.0212986.t001

There are multiple formatting errors in Table 3. Please see the complete, correct table here.

Table 3. The risk of HPAI transmission jumps from one area to another, due to a compartment with one farm located in the DPPA.

\begin{tabular}{l|l}
\hline Quantity & Value \\
\hline Ratio $^{1)}$ & $0.57(0.46-0.69)$ \\
\hline 'Single-event' transmission probability ${ }^{2)}$ & $1.110^{-6}-0.010$ \\
\hline 'Triple-event' transmission probability ${ }^{2)}$ & $1.210^{-7}-8.910^{-5}$ \\
\hline
\end{tabular}

\section{OPEn ACCESS}

Citation: The PLOS ONEStaff (2019) Correction: Risk of poultry compartments for transmission of Highly Pathogenic Avian Influenza. PLoS ONE 14(2): e0212986. https://doi.org/10.1371/journal. pone.0212986

Published: February 22, 2019

Copyright: @ 2019 The PLOS ONE Staff. This is an open access article distributed under the terms of the Creative Commons Attribution License, which permits unrestricted use, distribution, and reproduction in any medium, provided the original author and source are credited.

\footnotetext{
1) The risk of jumps is expressed as mean ratio of 'triple-event' transmission via the compartment and 'single-event' transmission by neighbourhood transmission (the 5\%-95\% interval given between brackets).

${ }^{2)}$ The range (min-max) of the probability for 'single-event' transmission and for 'triple-event' transmission are added.
}

https://doi.org/10.1371/journal.pone.0212986.t002

There are multiple formatting errors in Table 4. Please see the complete, correct table here. The publisher apologizes for these errors. 
Table 4. The risk of HPAI transmission jumps from one area to another, due to a compartment with one farm located in the DPPA and with transport of animals (chicken) and eggs.

\begin{tabular}{|c|c|}
\hline Quantity & Value \\
\hline Ratio $^{1)}$ & $0.62(0.46-0.78)$ \\
\hline 'Single-event' transmission probability ${ }^{2)}$ & $1.110^{-6}-0.0020$ \\
\hline 'Triple-event' transmission probability ${ }^{2)}$ & $1.210^{-7}-1.210^{-4}$ \\
\hline
\end{tabular}

1) The risk of jumps is expressed as mean ratio of 'triple-event' transmission via the compartment and 'single-event' transmission by neighbourhood transmission (the 5\%-95\% interval given between brackets).

2) The range (min-max) of the probability for 'single-event' transmission and for 'triple-event' transmission are added.

https://doi.org/10.1371/journal.pone.0212986.t003

\section{Reference}

1. Hagenaars TJ, Boender GJ, Bergevoet RHM, van Roermund HJW (2018) Risk of poultry compartments for transmission of Highly Pathogenic Avian Influenza. PLoS ONE 13(11): e0207076. https://doi.org/ 10.1371/journal.pone.0207076 PMID: 30485292 